\title{
Total Hip Arthroplasty Surgical Approach Does Not Alter Postoperative Gait Mechanics One Year After Surgery
}

\author{
Robin M. Queen, PhD, J. Stephen Appleton, MD, Robert J. Butler, PT, PhD, \\ Erik T. Newman, MD, Scott S. Kelley, MD, David E. Attarian, MD, \\ Michael P. Bolognesi, MD
}

Objective: To investigate the differences in gait biomechanics on the basis of surgical approach 1 year after surgery.

Design: This was a descriptive laboratory study to investigate the side-to-side differences in walking mechanics at a self-selected walking speed as well as a functional assessment 1 year after total hip arthroplasty (THA). Temporospatial, kinetic, and kinematic data as well as functional outcomes were collected. Two-way analysis of variance was used to assess for between-group differences and limb-to-limb asymmetries.

Setting: A controlled laboratory study.

Participants: This study examined 35 patients with primary, unilateral THA. The THA surgical approaches that were used in these patients included 12 direct lateral, 18 posterior, and 11 anterolateral. All the patients were assessed 1 year after THA. Patients were excluded from the study if they had contralateral hip pain or pathology, or any prior lower extremity total joint replacements.

Main Outcome Measurements: Three-dimensional lower extremity kinematics and kinetics as well as spatiotemporal variables were collected. In addition, a series of physical performance measures were collected.

Results: No main effects for the physical performance measures or biomechanical variables were observed among the approach groups. Significant limb-to-limb asymmetries were observed among all the patients, with decreased sagittal plane range of motion, peak extension, and peak vertical ground reaction forces on the operative side.

Conclusion: The results of this study indicated that no significant differences existed among the different surgical approach groups for any study variable. However, 1 year after THA, the patients demonstrated asymmetric gait patterns regardless of surgical approach, which indicated the potential need for continued intervention through physical therapy to regain normal side-to-side symmetry after THA.

PM R 2013; $: 1-6$

\section{INTRODUCTION}

Total hip arthroplasty (THA) is performed annually for more than a quarter of a million patients in the United States alone [1]. In the past half-century, there have been significant improvements in technique and implant technologies. Despite these advances, debate remains as to the superiority of any particular surgical approach. The ideal approach would minimize instability, pain, and postoperative weakness while maximizing patients' return to normal function of the replaced hip.

Several approaches to the hip are used by arthroplasty surgeons. Advocates of the posterior $(\mathrm{P})$ approach tout its relative technical ease and argue that maintenance of the integrity of the hip abductors helps to avoid postoperative abductor weakness and limp. However, this may come at the cost of increased incidence of hip instability and injury to the sciatic nerve during the surgical dissection [2-4]. The direct lateral (DL), or Hardinge, (For the sake of consistency, discussion of prior approach-related research will use the

R.M.Q. Department of Orthopaedic Surgery, Duke University Medical Center, Durham, NC; Michael W. Krzyzewski Human Performance Lab, 102 Finch Yeager Building, DUMC 3435, Duke University Medical Center, Durham, NC 27710. Address correspondence to: R.M. Q.; e-mail: robin.queen@duke.edu Disclosure: nothing to disclose

J.S.A. Department of Orthopaedic Surgery, Duke University Medical Center, Durham, NC Disclosure: nothing to disclose

R.J.B. Michael W. Krzyzewski Human Performance Lab, Duke University Medical Center, Durham, NC; Division of Physical Therapy, Department of Community and Family Medicine, Duke University Medical Center, Durham, NC

Disclosure: nothing to disclose

E.T.N. Department of Orthopaedic Surgery, Duke University Medical Center, Durham, NC Disclosure: nothing to disclose

S.S.K. Department of Orthopaedic Surgery, Duke University Medical Center, Durham, NC Disclosure: nothing to disclose

D.E.A. Department of Orthopaedic Surgery, Duke University Medical Center, Durham, NC Disclosure: nothing to disclose

M.P.B. Department of Orthopaedic Surgery, Duke University Medical Center, Durham, NC Disclosure: nothing to disclose

Research support: Orthopaedic Research and Education Foundation.

Submitted for publication February 6, 2013; accepted September 11, 2013. 
terminology used by the present study, eg, the Hardinge and Rottinger approaches will be described as direct lateral and anterolateral, respectively, regardless of the terms used in previous publications) approach allows excellent acetabular exposure, improved cup positioning, and, consequently, a decreased risk of postoperative instability and dislocation $[5,6]$. Some researchers maintain that the DL approach is associated with a relatively high risk of abductor weakness and postoperative limp secondary to gluteus medius violation and potential injury to the superior gluteal nerve [6-8]. Prospective comparative [9] and systematic review [5] data, however, do not support a difference between the DL and $\mathrm{P}$ approaches with respect to these adverse outcomes. The anterolateral (AL), or Rottinger, approach, as with the DL approach, allows for maintenance of posterior capsular attachments and short external rotators, which reduces postoperative instability while maintaining abductor integrity [10-12]. However, anterior approaches to the hip, possibly including the AL approach, have been associated with increased rates of lateral femoral cutaneous nerve injury [13] and fractures of the greater trochanter and femur $[14,15]$

Postoperative gait characteristics after THA have been the subject of a number of previous studies, many of which have demonstrated persistent postoperative biomechanical abnormalities at the operative hip [16-18]. However, there are few studies that directly compare the effect of the surgical approach on postoperative gait characteristics after THA. Data concerning early (6 weeks) postoperative functional differences among the approaches are mixed. The direct anterior approach has been associated with more symmetric single limb support and step length than the DL approach [19], but other studies have found no such early differences in temporospatial or biomechanical variables between the DL and P groups [20] or among the AL, DL, and P groups [21]. Several studies have also assessed temporospatial gait variables in different approach groups at 4-6 months after surgery. Lugade et al [19], when comparing direct anterior and DL groups, and Maffiuletti et al [22], when comparing direct anterior and $\mathrm{P}$ groups, found no differences between groups in temporospatial variables, such as step length and single limb support time. Madsen et al [23], when studying patients who had the DL and P approaches at 6 months after surgery, were also unable to identify temporospatial differences between the groups, but did report more normal hip flexion and extension in the P group compared with the DL group.

Gait analysis data that compare medium-term postoperative function after the different surgical approaches are needed. The current study focused on the effects of 3 common surgical approaches: AL, DL, and P, on gait symmetry 1 year after surgery. It was hypothesized that patients would demonstrate side-to-side asymmetries but that no significant temporospatial or biomechanical differences would exist among the groups.

\section{METHODS}

A total of 35 subjects (12 with the DL approach, 18 with the $\mathrm{P}$ approach, and 11 with the AL approach) participated in this study. All the subjects were more than 35 years old and were tested approximately 12 months after their primary unilateral THA. The patients were placed in a surgical approach group based on their treating physician because each physician had a preferred surgical approach. Patients were excluded from the study if they had a history of previous lower extremity total joint replacement, contralateral hip pain, or joint degeneration, or any history of neurologic disorders. Subjects undergoing THA were recruited from the orthopedic clinics at the university medical center. Informed consent approved by the institutional review board was signed by each subject before study initiation.

The 3 surgical approaches that were examined were the standard posterior (P), the DL (or modified Hardinge), and the AL (or modified Watson-Jones or Rottinger). The details of the 3 surgical approaches used during the THA for these patients have been previously described by Queen et al [21]. After surgery, all the subjects were given a set of exercises to complete at home when they were released from the hospital; however, no additional postoperative physical therapy was prescribed for any of the study patients. This is the standard postoperative rehabilitation course after THA at our institution. For the gait analysis, the subjects wore formfitting shorts and walked barefoot, thereby avoiding any effects associated with footwear. Reflective markers were placed, by a single tester, using previously reported methods $[21,24]$. Each subject was asked to stand with his or her feet shoulder-width apart within the capture volume for a standing trial to identify the hip joint center. The data collection methods used in this study have been previously used in patients with total hip replacement and have been reported previously by this research team $[21,24]$. The kinematic data were sampled at $120 \mathrm{~Hz}$, whereas the ground reaction force (GRF) data were collected at $1200 \mathrm{~Hz}$. All the subjects walked $10 \mathrm{~m}$ at a self-selected speed during 7 walking trials.

Height, weight, age, Harris Hip Score (HHS), and surgical approach were recorded for each patient. The following variables were analyzed to determine whether any significant differences existed among the approach groups or between the surgical and nonsurgical limbs: stance time, step length, stride length, swing time, peak weight acceptance, and peak propulsion vertical GRF (vGRF), peak hip extension angle, sagittal plane hip range of motion, hip flexion-extension angle at heel strike, peak hip abduction angle, and hip abduction-adduction angle at heel strike. Step and stride length were normalized to each subject's standing height; stance, step, and swing time were normalized as a percentage of the gait cycle, whereas GRF was normalized to body weight. All of these measures have been previously reported in this patient population by Queen et al $[21,24,25]$. In 
addition to the biomechanical measures of interest, the following functional measures, Timed Up and Go Test (TUG), timed Sit-to-Stand Test, and self-selected walking speed, as well as the Harris Hip Score (HHS), Activity Scale for Arthroplasty Patients (ASAP), and University of California, Los Angeles scores (UCLA score) were collected for each subject to compare among the 3 surgical approach groups.

A $1 \times 3$ analysis of variance (ANOVA) was used to compare the HHS, ASAP, UCLA score, and the functional outcomes 1 year after THA. In addition, a $2 \times 3$ (limb-by-approach) ANOVA was used for analysis of the gait variables. The limb was defined on the basis of operative versus nonoperative side, whereas approach was defined as P, DL, or AL. A separate ANOVA $(\alpha=0.05)$ was completed for each study variable of interest. Tukey post hoc testing was completed on any variables that were statistically different.

\section{RESULTS}

No significant demographic differences existed among the approach groups in age, height, or weight by l-way ANOVA analysis (Table 1). In addition, no statistically significant differences existed in HHS, ASAP, UCLA scores, Timed Up and Go Test, Sit-to-Stand Test, or self-selected walking speed across the groups. No statistically significant differences existed among the surgical approaches for peak hip extension angle, peak hip extension moment, peak hip adduction angle during the stance phase, or peak hip adduction moment during weight acceptance. In addition, the peak vGRF, hip range of motion, hip angle at heel strike in the sagittal and frontal planes, stance time, swing time, and step length were not significantly different among the approach groups.

A significant limb-by-approach interaction was observed for peak hip adduction moment during the propulsion phase of gait $(P<.01)$ (Figure 1$)$. Post hoc analysis showed that this interaction was driven by differences between the AL group and the 2 other groups. The patients in the AL group demonstrated increased adduction moment at the operative compared with the nonoperative hip, whereas patients in the

Table 1. Demographic data and functional outcome comparisons among groups (mean (SD)**

\begin{tabular}{lccr}
\hline & Direct Lateral & Posterior & Anterolateral \\
\hline Age,y & $61.25 \pm 6.93$ & $55.83 \pm 8.13$ & $57.27 \pm 10.65$ \\
Weight, $\mathrm{kg}$ & $81.96 \pm 19.96$ & $82.74 \pm 19.32$ & $81.19 \pm 18.77$ \\
Height, $\mathrm{m}$ & $1.70 \pm 0.11$ & $1.73 \pm 0.09$ & $1.69 \pm 0.09$ \\
TUG, s & $6.26 \pm 0.75$ & $5.58 \pm 1.02$ & $5.64 \pm 1.11$ \\
STS, $s$ & $15.08 \pm 2.75$ & $17.67 \pm 6.52$ & $18.36 \pm 5.50$ \\
HHS & $94.00 \pm 3.02$ & $91.78 \pm 6.03$ & $93.30 \pm 3.83$ \\
UCLA & $7.17 \pm 1.40$ & $7.22 \pm 2.05$ & $6.30 \pm 1.42$ \\
ASAP & $33.58 \pm 9.10$ & $34.78 \pm 7.23$ & $32.63 \pm 6.92$ \\
SSWS, m/s & $1.28 \pm 0.11$ & $1.36 \pm 0.21$ & $1.40 \pm 0.24$ \\
\hline
\end{tabular}

$\mathrm{SD}=$ standard deviation; TUG = Timed Up and Go Test; STS = Sit to Stand Test; HHS = Harris Hip Score; UCLA = University of California Los Angeles score; ASAP = Activity Scale for Arthroplasty Patients; SSWS, self-selected walking speed.

*No statistically significant differences among the groups.

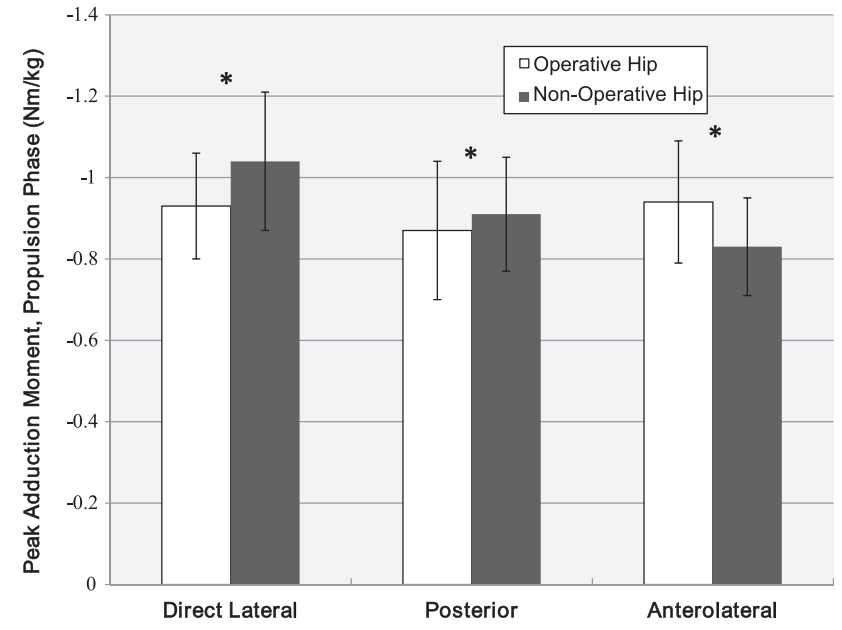

Figure 1. Peak hip adduction moment during the propulsion phase at the operative and nonoperative hips, by approach group. *Significant limb-by-approach interaction $(P<.01)$.

$\mathrm{P}$ and DL groups demonstrated decreased operative hip adduction moment. Operative and nonoperative limb adduction moments during the propulsion phase for the $\mathrm{AL}$, $\mathrm{P}$, and DL groups were mean (SD) $-0.94 \pm 0.15 \mathrm{Nm} / \mathrm{kg}$ and $-0.83 \pm 0.12 \mathrm{Nm} / \mathrm{kg} ;-0.87 \pm 0.17 \mathrm{Nm} / \mathrm{kg}$ and -0.91 $\pm 0.14 \mathrm{Nm} / \mathrm{kg}$; and $-0.93 \pm 0.13 \mathrm{Nm} / \mathrm{kg}$ and $-1.04 \pm 0.17$ $\mathrm{Nm} / \mathrm{kg}$, respectively. A number of variables differed significantly between limbs, independent of the surgical approach (Table 2). Among all the patients, hip range of motion in the sagittal plane was decreased on the operative side $\left(35.81^{\circ}\right.$ $\left.\pm 5.77^{\circ}\right)$ compared with the nonoperative side $\left(39.44^{\circ} \pm\right.$ $\left.6.08^{\circ}\right)(P<.001)$. An asymmetry also existed with respect to peak hip extension, with a decreased angle observed for the operative $\left(-5.38^{\circ} \pm 7.94^{\circ}\right)$ compared with the nonoperative $\left(-8.31^{\circ} \pm 8.00^{\circ}\right) \operatorname{limb}(P<.001)$. In addition, peak vGRF during weight acceptance and propulsion phases $(P<.05)$ were decreased on the operative side, regardless of approach.

\section{DISCUSSION}

Debate regarding the surgical approach for THA is ongoing, with risks and benefits of each approach described in the literature. However, there remains no clear consensus regarding the best surgical approach for primary THA. The choice of approach remains dependent primarily on surgeon preference, which frequently is influenced by training history and personal experience. As noted above, several prior studies have been performed to evaluate gait differences among the surgical approaches $[19,21,23]$. To our knowledge, this is the first gait biomechanics study to evaluate these 3 surgical approaches one year following surgery. No main effects differences in temporospatial or biomechanical variables were observed among the approach groups. However, a significant limb-by-approach interaction existed 
Table 2. Biomechanical comparisons between limbs, all patients (mean (SD))

\begin{tabular}{lrr}
\hline & Operative & Nonoperative \\
\hline Hip & & \\
Sagittal ROM, degree & $35.81 \pm 5.97^{*}$ & $39.44 \pm 6.08^{*}$ \\
Frontal ROM, degree & $9.72 \pm 2.94$ & $9.91 \pm 2.90$ \\
Sagittal angle at HS, degree & $30.30 \pm 7.38$ & $30.82 \pm 7.57$ \\
Frontal angle at HS, degree & $3.22 \pm 3.81$ & $1.87 \pm 4.12$ \\
Peak hip & & \\
Ext (-) angle, degree & $-5.38 \pm 7.94^{*}$ & $-8.31 \pm 8.00^{*}$ \\
Ext (-) mom, Nm/kg & $-0.98 \pm 0.29$ & $-1.02 \pm 0.30$ \\
Add (+) angle, degree & $9.27 \pm 3.62$ & $8.29 \pm 3.76$ \\
First add (-) mom, Nm/kg & $-0.97 \pm 0.17$ & $-1.00 \pm 0.19$ \\
Second add (-) & $-0.91 \pm 0.15$ & $-0.93 \pm 0.16$ \\
mom, Nm/kg & & \\
Peak hip & & \\
Weight acceptance (BW) & $1.11 \pm 0.13^{\dagger}$ & $1.13 \pm 0.14^{\dagger}$ \\
Propulsion (BW) & $1.11 \pm 0.10^{\dagger}$ & $1.12 \pm 0.11^{\dagger}$ \\
Stance time, s & $59.33 \pm 1.64$ & $59.37 \pm 1.93$ \\
Swing time, s & $40.60 \pm 1.51$ & $40.60 \pm 1.91$ \\
NORM step length, $\mathrm{m}$ & $0.67 \pm 0.09$ & $0.67 \pm 0.09$
\end{tabular}

$\mathrm{SD}=$ standard deviation; $\mathrm{ROM}=$ range of motion; $\mathrm{HS}=$ heel strike; $\mathrm{Ext}=$ extension; $\mathrm{mom}=$ moment; $\mathrm{Add}=$ adduction; $\mathrm{BW}=$ body weight; NORM = normalized to subject's standing height.

*Significant difference between operative and nonoperative limbs, all patients $(P<.001)$.

${ }^{\dagger}$ Significant difference between operative and nonoperative limbs, all patients $(P<.05)$.

for peak hip adduction moment during the propulsion phase of gait. Although the patients in the $\mathrm{P}$ and $\mathrm{DL}$ groups demonstrated decreased adduction moments at the operative compared with the nonoperative hip, the opposite was true for patients in the AL group.

The clinical significance of the interaction for the frontal plane moment is unclear. The effects of the AL approach on the hip's ability to generate adduction forces during the act of active hip extension does not seem to correlate with the potential damage to the structures in the anterior or anterolateral area of the hip from an anatomic standpoint. The relative increase in adductor moment at the operative hip in patients in the AL group may have corresponded with pelvic drop during the propulsion phase. This could be attributable to weakness of the abductor mechanism. Klausmeier et al [26] (with $\mathrm{n}=23$ patients with THA) demonstrated that patients having undergone the AL approach experienced slower recovery of hip abductor function at 6 weeks after surgery compared with the patients who had the direct anterior approach, perhaps suggesting that some degree of abductor trauma may occur in the AL approach. As noted above, prior results would suggest that any biomechanical abnormality that results from abductor weakness might have been most evident among patients in the DL group [6-8,27].

It also is conceivable that, given the absence of preoperative gait assessment data and the relatively small sample size in the present study, the adduction moment limbby-approach interaction finding was the result of persistence of preoperative between-group differences. However, no other between-group temporospatial or biomechanical differences existed. Likewise, no between-group differences were observed in self-report questionnaire scores (HHS, ASAP, and UCLA scores) or in physical performance testing (Timed Up and Go Test, Sit-to-Stand Test, self-selected walking speed), which suggests substantial clinical parity among groups. This is in line with numerous previous studies that found no differences in temporospatial variables among the approach groups at earlier postoperative time points [19-22].

Multiple side-to-side asymmetries independent of surgical approach were observed, which is in contrast to previous biomechanical data that demonstrated higher rates of asymmetry among patients who had the DL approach compared with those who had the $\mathrm{P}$ approach [23]. In addition to asymmetric GRFs, the patients walked with decreased sagittal plane (flexion-extension) range of motion on the operative side compared with the nonoperative side. A similar asymmetry existed with respect to peak hip extension, which suggests that the limitation in operative limb sagittal range of motion was driven, at least in part, by incomplete extension. It is possible that this was the result of alterations to the soft-tissue envelope, including joint capsule or extensor mechanism. Although the peak joint angle difference is less than $10^{\circ}$ for combined peak flexion and peak extension, the overall difference of approximately $8^{\circ}$ in hip range of motion between the surgical and nonsurgical limbs during stance would likely have clinical relevance due to the large effect size $(>1.0)$. Madsen et al [23], in comparing DL $(\mathrm{n}=10)$ and $\mathrm{P}(\mathrm{n}=10)$ groups with healthy controls $(n=9)$, also reported decreased sagittal motion in postoperative patients, with the greatest flexion and extension limitations observed in the DL group. The present study found that deficiencies in hip extension persisted for all 3 approaches at 1 year after surgery.

The vGRFs were noted to be significantly decreased on the operative limb, independent of approach, during both weight acceptance and propulsion. The postoperative patients studied may have continued to guard the replaced joint during ambulation, even at 1 year after surgery. Indeed, previous reports have demonstrated decreased vGRFs and altered temporal loading patterns in patients with postoperative THA compared with subjects' nonoperative hip and the normal controls [28,29]. Lugade et al [19] found that the asymmetric vGRF loading observed before surgery in direct anterior and DL groups persisted at 6 weeks after surgery but was no longer present in either group at 16 weeks. Although continued asymmetric loading was noted in all 3 surgical groups, it could be concluded that the relatively small asymmetries observed here may not be clinically relevant, even if they are statistically so.

In contrast to the results published by Lugade et al [19], no side-to-side asymmetries were observed among any of the postoperative groups with respect to temporospatial variables (stance time, swing time, step length). The followup period of the present study was substantially longer, 
12-month versus the 6-month data that were reported by Lugade et al [19], which suggests that gait asymmetries may continue to improve beyond 6 months and potentially beyond 1 year for all 3 of the approaches studied here. Although these measures of gait asymmetry appear to be improving by 1 year after THA, certain asymmetries did persist at 1 year after surgery, consistent with previous studies that, as outlined above, have demonstrated failure of those undergoing THA to return to completely normal gait after surgery $[16,19,22,23]$. These differences, however, were independent of surgical approach, which suggests that the procedure itself was the causative factor rather than the choice of surgical approach.

Limitations of the current study include its nonrandomized design, although no differences in baseline demographics existed between groups, and we can think of no reasons for selection bias with respect to patients' choice of surgeon. Perhaps more problematic was the absence of preoperative gait data; it is possible that preoperative between-group differences influenced the postoperative results in immeasurable ways. In addition, each surgical approach was performed by a different surgeon. Distinct differences in technique, apart from surgical approach, used by these individual surgeons could potentially influence postoperative results. It should be considered, however, that each of these surgeons is highly skilled in his particular approach and in hip arthroplasty in general. This expertise and specialization likely ensured consistency and minimized outliers. Finally, we did not assess hip muscle strength for any of these subjects. Muscle strength could play a role in explaining the minor statistical differences that are seen among these surgical approach groups.

In conclusion, 3 surgical approaches were compared at 1 year after surgery, and no between-group differences were observed. A significant approach-by-limb interaction did exist for hip adduction moment during propulsion; however, this difference does not appear to be clinically relevant. In addition, differences between the operative and nonoperative hips were observed with respect to range of motion in the sagittal plane and peak vGRF during both weight acceptance and propulsion. We would hypothesize that, although these differences may marginally diminish beyond the 1-year postoperative mark, they would likely remain present throughout any future data points. It appears that the improvements (and continued asymmetries) in gait mechanics after THA are the result of the act of joint replacement itself than the specific surgical approach used.

\section{ACKNOWLEDGMENT}

We thank the Orthopaedic Research and Education Foundation for funding this study.

\section{REFERENCES}

1. Number of all-listed procedures from discharges from short-stay hospitals by procedure category and age: United States 2009. Available at http://www.cdc.gov/nchs/data/nhds/4procedures/2009pro4_number procedureage.pdf. Accessed December 28, 2012.

2. Masonis J, Bourne R. Surgical approach, abductor function, and total hip arthroplasty dislocation. Clin Orthop Related Res 2002;405:46-53.

3. Ritter M, Harty L, Keating M, Faris P, Medin J. A clinical comparison of the anterolateral and the posterolateral approaches to the hip. Clin Orthop Related Res 2001;385:95-99.

4. Woo R, Morrey B. Dislocations after total hip arthroplasty. J Bone Joint Surg Am 1982;64:1295-1305.

5. Jolles B, Bogoch E. Posterior versus lateral surgical approach for total hip arthroplastyin adults with osteoarthritis. Cochrane Database Syst Rev 2004;(1):CD003828.

6. Mulliken BD, Rorabeck CH, Bourne RB, Nayak N. A modified direct lateral approach in total hip arthroplasty: A comprehensive review. J Arthroplasty 1998;13:737-747.

7. Baker A, Bitounis VC. Abductor function after total hip replacement: An electromyographic and clinical review. J Bone Joint Surg Br 1989; $71: 47-50$

8. Callaghan JJ, Dysart SH, Savory CF, Hopkinson WJ. Assessing the results of hip replacement. A comparison of five different rating systems. J Bone Joint Surg Br 1990;72:1008-1009.

9. Downing N, Clark D, Hutchinson J, Clolclough K, Howard P. Hip abductor strength following total hip arthroplasty: A prospective comparison of the posterior and lateral approach in 100 patients. Acta Orthop Scand 2001;72:215-220.

10. Bertin $K C$, Rottinger $H$. Anterolateral mini-incision hip replacement surgery: A modified Watson-Jones approach. Clin Orthop Relat Res 2004:429:248-255.

11. Hansen BJ, Hallows RK, Kelley SS. The Rottinger approach for total hip arthroplasty: Technique and review of the literature. Curr Rev Musculoskelet Med 2011:4:132-138.

12. Muller M, Tohtz S, Springer $I$, Dewey M, Perka C. Randomized controlled trial of abductor muscle damage in relation to the surgical approach for primary total hip replacement: Minimally invasive anterolateral versus modified direct lateral approach. Arch Orthop Trauma Surg 2011;131:179-189.

13. Goulding K, Beaule PE, Kim PR, Fazekas A. Incidence of lateral femoral cutaneous nerve neuropraxia after anterior approach hip arthroplasty. Clin Orthop Relat Res 2010;468:2397-2404.

14. Jewett $B A$, Collis DK. High complication rate with anterior total hip arthroplasties on a fracture table. Clin Orthop Relat Res 2011;469:503-507.

15. Matta JM, Shahrdar C, Ferguson T. Single-incision anterior approach for total hip arthroplasty on an orthopaedic table. Clin Orthop Relat Res 2005;441:115-124.

16. Perron $M$, Malouin F, Moffet $H$, McFadyen BJ. Three-dimensional gait analysis in women with a total hip arthroplasty. Clin Biomech (Bristol, Avon) 2000;15:504-515.

17. Foucher KC, Hurwitz DE, Wimmer MA. Preoperative gait adaptations persist one year after surgery in clinically well-functioning total hip replacement patients. J Biomech 2007;40:3432-3437.

18. Kyriazis $V$, Rigas $C$. Temporal gait analysis of hip osteoarthritic patients operated with cementless hip replacement. Clin Biomech (Bristol, Avon) 2002;17:318-321.

19. Lugade V, Wu A, Jewett B, Collis D, Chou LS. Gait asymmetry following an anterior and anterolateral approach to total hip arthroplasty. Clin Biomech (Bristol, Avon) 2010;25:675-680.

20. Meneghini RM, Smits SA, Swinford RR, Bahamonde RE. A randomized, prospective study of 3 minimally invasive surgical approaches in total hip arthroplasty: Comprehensive gait analysis. J Arthroplasty 2008; 23(Suppl 1):68-73. 
21. Queen RM, Butler RJ, Watters TS, Kelley SS, Attarian DE, Bolognesi MP. The effect of total hip arthroplasty surgical approach on postoperative gait mechanics. J Arthroplasty 2011;26(Suppl): 66-71.

22. Maffiuletti NA, Impellizzeri FM, Widler $\mathrm{K}$, et al. Spatiotemporal parameters of gait after total hip replacement: Anterior versus posterior approach. Orthop Clin North Am 2009;40:407-415.

23. Madsen MS, Ritter MA, Morris HH, et al. The effect of total hip arthroplasty surgical approach on gait. J Orthop Res 2004;22: 44-50.

24. Queen RM, Watters TS, Abbey AN, Sabesan VJ, Vail TP, Bolognesi MP. Gait symmetry: A comparison of hip resurfacing and jumbo head total hip arthroplasty patients. J Arthroplasty 2011;26: 680-685.
25. Queen RM, Newman ET, Abbey AN, Vail TP, Bolognesi MP. Stair ascending and descending in hip resurfacing and large head total hip arthroplasty patients. J Arthroplasty 2013;28:684-689.

26. Klausmeier V, Lugade V, Jewett BA, Collis DK, Chou LS. Is there faster recovery with an anterior or anterolateral THA? A pilot study. Clin Orthop Relat Res 2010;468:533-541.

27. Moskal JT, Mann JW III. A modified direct lateral approach for primary and revision total hip arthroplasty. A prospective analysis of 453 cases. J Arthroplasty 1996;11:255-266.

28. McCrory JL, White SC, Lifeso RM. Vertical ground reaction forces: Objective measures of gait following hip arthroplasty. Gait Posture 2001;14:104-109.

29. Long WT, Dorr LD, Healy B, Perry J. Functional recovery of noncemented total hip arthroplasty. Clin Orthop Relat Res 1993;288:73-77. 